\title{
Effect of Solvent and Temperature on the Anodic Oxide Films Formed on Nb in 0.1 M EDTA (Sodium Salt): Scanned Electron Micrograph Studies
}

\author{
V. JEEVANAJYOTHI ${ }^{1 *}$ and CH. ANJANEYULU ${ }^{2}$ \\ ${ }^{1}$ Department of Chemistry, RBVRR Women's College, Narayanaguda, Hyderabad, India \\ ${ }^{2}$ Department of Chemistry, Osmania University, India \\ jeevana.j@rediffmail.com
}

Received 30 May 2018 / Accepted 20 June 2018

\begin{abstract}
Anodization of $\mathrm{Nb}$ in $0.1 \mathrm{M}$ EDTA (Sodium salt) has been carried out. Kinetics of anodic oxidation of $\mathrm{Nb}$ has been studied at a constant current density of $8 \mathrm{~mA} . \mathrm{cm}^{-2}$ and at room temperature. The plots of formation voltage $v s$. time, reciprocal capacitance $v s$. time and reciprocal capacitance $v s$. formation voltage were drawn. From these plots, formation rate, current efficiency and differential field were calculated. The Addition of solvent (Ethylene glycol) showed better kinetic results. For 20, 40, 60 and 80\% aquo-glycolic media, the dielectric constant values are low leading to the marked improvement in the kinetics. The surface morphology of the anodic films was also studied by scanning electron micrographs (SEM). Kinetics was also studied at different temperatures ranging from $273 \mathrm{~K}$ to $333 \mathrm{~K}$ at a constant current density of $8 \mathrm{~mA} \mathrm{~cm}$. $^{-2}$. It was observed that kinetic results were found to be increasing linearly with the decrease in temperature
\end{abstract}

Keywords: Anodization, Formation rate, Current efficiency, Differential field, Nb, EDTA (Sodium salt), Temperature

\section{Introduction}

Metals such as niobium, zirconium and its alloys, tantalum, titanium etc., can be anodized to give thin, uniform and relatively flaw free oxide films, which are stable chemically and mechanically. Anodic oxide films formed on Niobium are useful in the field of electrical and electronic components (capacitors, resistors, diodes, photoelectric devises etc.), corrosion protection and for decorative purposes. Several other applications have been investigated by others $^{1-5}$.

In the present work, the kinetics of anodic oxidation of niobium in aqueous solution of 0.1 M EDTA (Sodium salt) and aquo-glycolic media in various proportions (v/v) of waterethylene glycol mixtures ranging from 0 to $80 \%$ ethylene glycol were studied. Scanning electron microscopic studies was also carried out to observe the changes in the surface morphology of the anodic films formed. An attempt was also made to study the kinetics at various temperatures ranging from $273 \mathrm{~K}$ to $333 \mathrm{~K}$ at a constant current density of $8 \mathrm{~mA} \mathrm{~cm}{ }^{-2}$. 


\section{Experimental}

Niobium was of $99.5 \%$ purity, supplied in the form of annealed sheet by NFC, Hyderabad as gift samples. In the present work, the foil samples used were cut with the aid of a punch into flag shaped specimens of $1 \mathrm{~cm}^{2}$ working area on both side and $2 \mathrm{~cm}$ long tag. The chemical polishing mixture for niobium is 5:5:1.5 by volume $53 \% \mathrm{HNO}_{3}, 49 \% \mathrm{HF}$ and $98 \% \mathrm{H}_{2} \mathrm{SO}_{4}$. For anodizing, a closed shell of $200 \mathrm{~mL}$ capacity was used. The cathode used was a platinum foil of $20 \mathrm{~cm}^{2}$ superficial area to make double layer capacitance as large as possible. Electrolytes used were 0.1 M EDTA (Sodium salt) in 20, 40, 60 and 80\% aquo-glycolic mixtures, the solvent being ethylene glycol. The temperatures used in the current study were ranging from $273 \mathrm{~K}$ to $333 \mathrm{~K}$. All the experiments were carried out at a constant current density of $8 \mathrm{~mA}$. $\mathrm{Cm}^{-2}$. The experimental procedure for the anodization is given elsewhere ${ }^{6}$. The kinetic results calculated are formation rate in $\mathrm{Vs}^{-1}$, current efficiency (n) $\%$ and differential fields of formation (FD) in $\mathrm{MV} \mathrm{cm}^{-1}$ from the conventional plots $\mathrm{V}$ vs. $\mathrm{t}, 1 / \mathrm{C}$ vs. $\mathrm{t}$ and $1 / \mathrm{C} v s . \mathrm{V}$.

The surface morphology of the anodic film was examined using scanning electron microscope (SEM). The SEM's were taken at IICT, CSIR and Hyderabad. The surface was thoroughly rinsed with distilled water and dried under infrared lamp and cleaned gently with fine tissue paper. The surface was coated with gold, palladium conducting material using evaporation technique and signal processed secondary electron images were taken from scanning electron microscope model SEM Hitachi- S520 at $10 \mathrm{KV}$, Hitachi instruments made in Japan (Oxford link ISIS- 300 UK)

\section{Results and Discussion}

\section{Solvent effect and SEM studies}

Anodization of niobium in $0.1 \mathrm{M}$ EDTA (Sodium salt) was performed by mixing various proportions of ethylene glycol to the aqueous solution (20,40,60 and 80\%). There was an improvement in the kinetics as given in Table 1. The relevant plots are shown in Figure 1 and Figure 2. Aquo-organic solutions aid in the formation of good oxide films and act as better electrolytic capacitors ${ }^{7}$. These facts support the current results obtained in aquoorganic mixtures of 0.1 M EDTA (sodium salt). It can be explained on the basis of decrease in the dielectric constant of the medium as shown in Table 2.

Table 1. Anodic films formed on niobium in 0.1 M EDTA (Sodium salt) both aqueous and aquo-glycolic-media

\begin{tabular}{|c|c|c|c|}
\hline Electrolyte & $\begin{array}{l}\text { Formation rate, } \\
\mathrm{dV} / \mathrm{dt}, \mathrm{V} \cdot \mathrm{s}^{-1}\end{array}$ & $\begin{array}{c}\text { Current } \\
\text { efficiency, } \eta, \%\end{array}$ & $\begin{array}{l}\text { Differential field, } \mathrm{F}_{\mathrm{D}} \\
\left(\mathrm{MV} \cdot \mathrm{cm}^{-1}\right)\end{array}$ \\
\hline 0.1 M EDTA(sodium salt) & 1.98 & 72.0 & 5.465 \\
\hline $\begin{array}{l}0.1 \mathrm{M} \text { EDTA(sodium salt) } \\
+20 \% \text { EG }\end{array}$ & 2.32 & 85.1 & 5.407 \\
\hline $\begin{array}{l}0.1 \mathrm{M} \text { EDTA(sodium salt) } \\
+40 \% \text { EG }\end{array}$ & 2.44 & 90.2 & 5.358 \\
\hline $\begin{array}{l}0.1 \mathrm{M} \text { EDTA(sodium salt) } \\
+60 \% \mathrm{EG}\end{array}$ & 2.53 & 94.3 & 5.321 \\
\hline $\begin{array}{l}0.1 \mathrm{M} \text { EDTA(sodium salt) } \\
+80 \% \text { EG }\end{array}$ & 2.60 & 97.2 & 5.295 \\
\hline
\end{tabular}

EDTA-Ethylene diamine tetra acetic acid EG-Ethylene glycol 


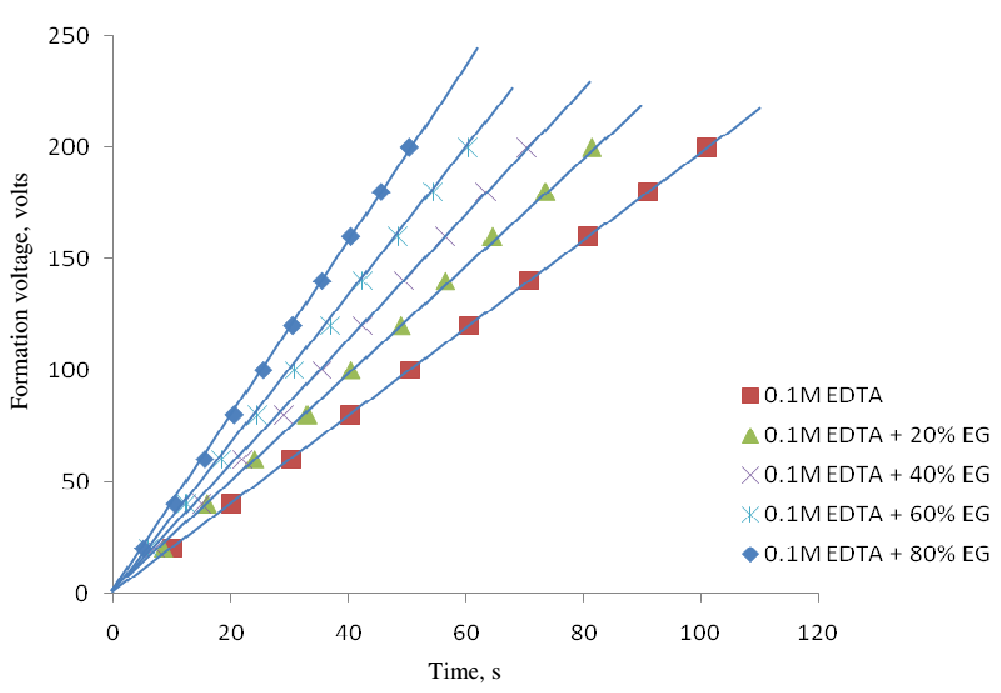

Figure 1. Plot of formation voltage as a function of time in aquo-glycolic solution

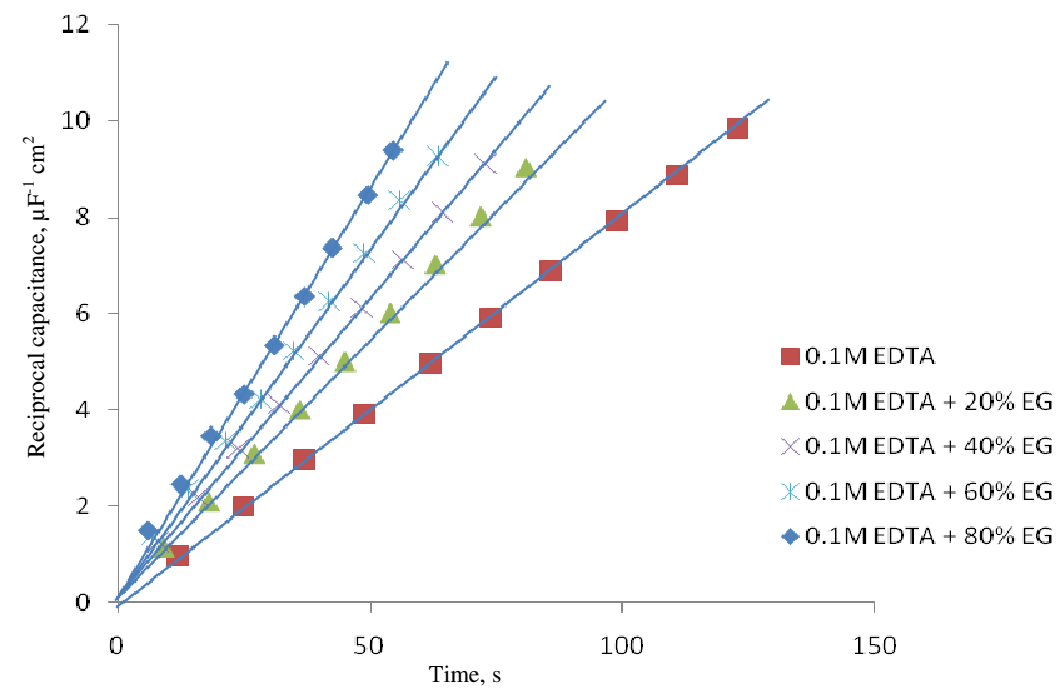

Figure 2. Plot of reciprocal capacitance as a function of time in aquo-glycolic solution

Table 2. Variation of dielectric constant as a function of time in aquo-glycolic solution

\begin{tabular}{ccccccc}
\hline EG , \% & 0 & 20 & 40 & 60 & 80 & 100 \\
\hline Dielectric constant & 80.0 & 72.8 & 69.2 & 57.8 & 43.2 & 37.7
\end{tabular}

In solutions of low dielectric constant there is less chance of ion-dipole interactions (solvent-ion interactions) which do not interfere in the oxide film formation. However the ions in the high dielectric constant solutions interact with oxide ions responsible for oxide film formation due to high solvation with water molecules. In such solutions, the kinetics is poor. The kinetics are better in low dielectric constant solutions for 20, 40, 60 and 80\% aquo-glycolic media, the dielectric constant values are low leading to a marked improvement in the kinetics. 


\section{Scanning electron micrographs}

Figure 3 and Figure 4 are the scanning electron micrographs of anodic films formed on niobium in aqueous and aquo-glycolic solutions of 0.1 M EDTA (Sodium salt)upto 200V.

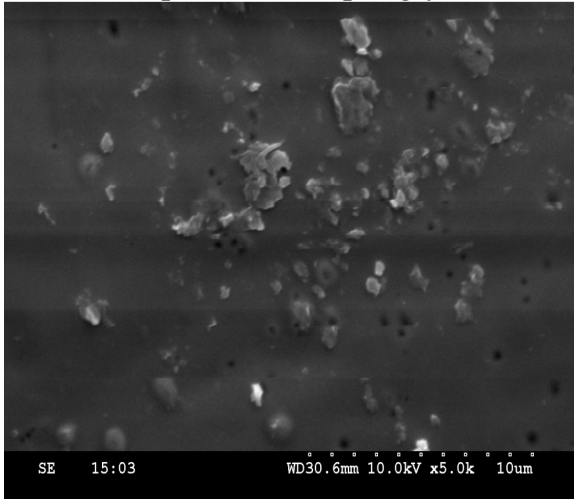

Figure 3. SEM of the film formed up to $220 \mathrm{~V}$ in $0.1 \mathrm{M}$ EDTA (Sodium salt) (aqueous medium) at room temperature (Magnification of the micrograph is X 5000)

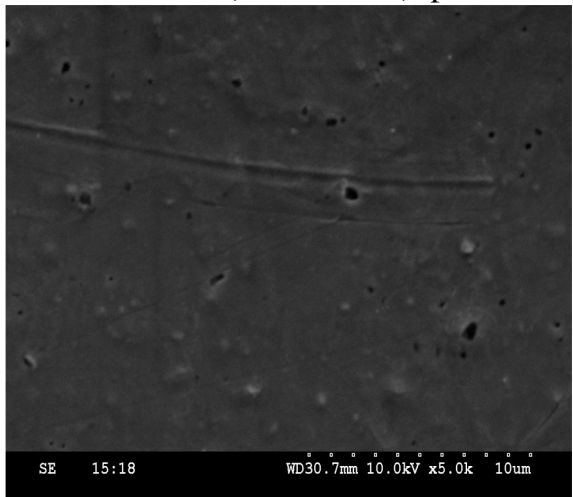

Figure 4. SEM of the film formed upto $220 \mathrm{~V}$ in $0.1 \mathrm{M}$ EDTA (Sodium salt) (glycolic medium) at room temperature (Magnification of the micrograph is X 5000)

When compared, the roughness and non-uniformity of the surface are decreased when electrolytic medium is changed from aqueous to aquo-glycolic medium (Figure 4) is observed to be smoother than the film formed in aqueous medium (Figure 3)

Vermilyea ${ }^{8}$ studied the formation of anodic films on tantalum in aqueous and nonaqueous ${ }^{9}$ solutions. He suggested that the composition of film depends on the solution in which it is formed. Nageshwarrao et al. ${ }^{10}$ observed a change in the dielectric constant of oxide films by changing the medium from aqueous to glycolic. Vermilyea ${ }^{9}$ reported that the optical thickness was smaller in the non-aqueous solutions and the increase in weight for a given charge passed could be as much as twice that expected. Moshashi Koyama ${ }^{11}$ carried out anodization of titanium in non-aqueous media and confirmed that the oxide film consists of double layers and suitable for electrolytic capacitors.

Wei Wei et al., ${ }^{12}$ reported the growth of layers by anodization of tantalum in a nonaqueous electrolyte consisting of an optimized glycerol/ethylene glycol mixture with the addition of $\mathrm{NH}_{4} \mathrm{~F}$. Schmidt et al., ${ }^{13}$ observed that the layers of $\mathrm{TiO}_{2}$ obtained in non-aqueous electrolytes are much adherent and uniform than those realized in aqueous media. Lavanya ${ }^{14}$ studied Anodization of zirconium-niobium-copper (ZNC) alloy in $0.1 \mathrm{M}$ mandelic acid and suggested that the effect of solvent (ethylene glycol) and added anionic impurities (sulphates, phosphates and carbonates) showed better kinetic results

Panasa Reddy et al., ${ }^{15}$ and Lavanya et al., ${ }^{16}$ also studied in trisodium citrate, $0.1 \mathrm{M}$ $\mathrm{KOH}$ (aquo-glycolic) respectively and found that the breakdown voltage was higher when anodized in ethylene glycol medium. This was also supported by other worker ${ }^{17}$. Shukla ${ }^{18}$ carried out the study of effect of aquo-glycolic media on anodization of zircaloy-4 in $0.1 \mathrm{M}$ sulphamic acid and found that the addition of solvent improved the kinetic results.

\section{Effect of temperature}

Anodizations were carried out on niobium at various temperatures ranging from $273 \mathrm{~K}$ to $333 \mathrm{~K}$ and at a constant current density of $8 \mathrm{~mA} \cdot \mathrm{cm}^{-2}$ in $0.1 \mathrm{M}$ EDTA (Sodium salt). 
The conventional plots were drawn as shown in the Figure 5 and Figure 6. From these plots, the kinetic results were calculated. It was observed that the formation rate, current efficiency, differential field and break down voltage were found to be increasing linearly with the decrease in temperature as shown in Table 3. The decrease in the field, formation rate and Breakdown voltage with the increase in temperature may be attributed to the decrease in the incorporation of anions into the film and may also be due to the dissolution of the film with the increase in temperature. Colton and Wood ${ }^{19}$ reported that the BDV was decreased with increase in temperature in the case of titanium oxide films and explained the observation on the basis of dissolution of the film. Bhaskarreddy et al. ${ }^{20}$ observed the similar trend of decreasing kinetic results with increase in temperature in $0.1 \mathrm{M}$ potassium tartrate. The same results were observed by other workers ${ }^{21-26}$.

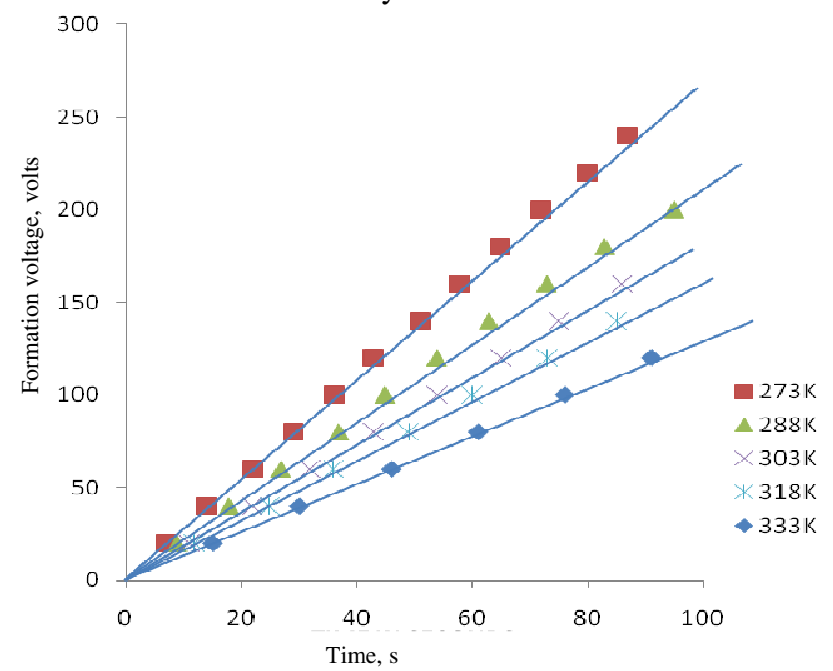

Figure 5. Plot of formation voltage as a function of time at different temperatures

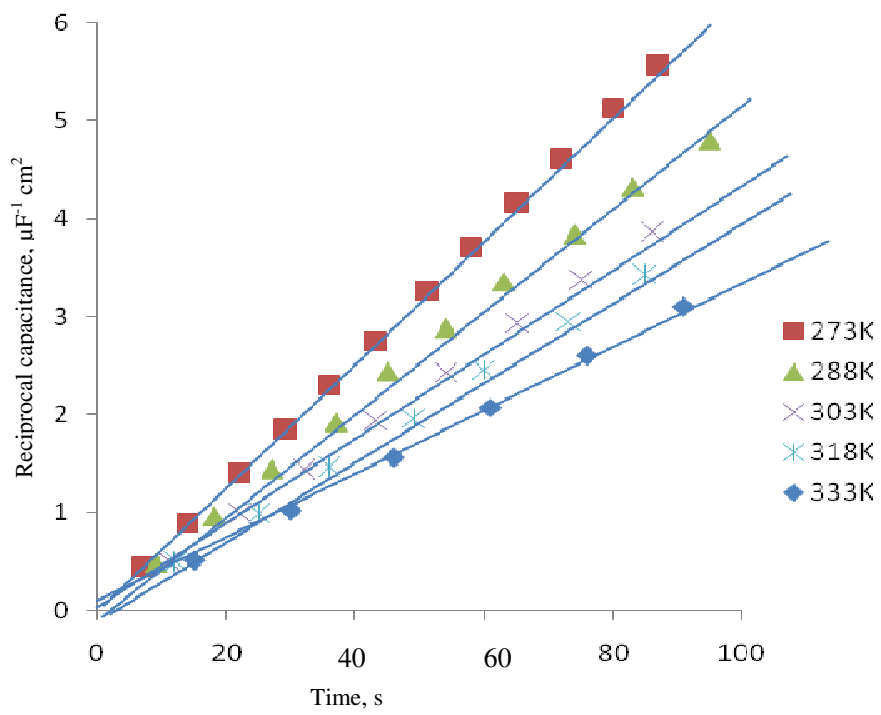

Figure 6. Plot of reciprocal capacitance as a function of time at different temperatures 
Table 3. Anodic films formed on niobium in $0.1 \mathrm{M}$ EDTA (Sodium salt) at different temperatures

\begin{tabular}{ccccc}
\hline $\begin{array}{c}\text { Temperature, } \\
\text { T, K }\end{array}$ & $\begin{array}{c}\text { Formation } \\
\text { rate, dV/dt, V.s }\end{array}$ & $\begin{array}{c}\text { Current } \\
\text { efficiency, } \eta, \%\end{array}$ & $\begin{array}{c}\text { Differential } \\
\text { field, } \mathrm{F}_{\mathrm{D}} \\
\left(\mathrm{MV} . \mathrm{cm}^{-1}\right)\end{array}$ & $\begin{array}{c}\text { Break down } \\
\text { voltage }\left(\mathrm{V}_{\mathrm{B}}\right), \\
\text { Volts }\end{array}$ \\
\hline 273 & 2.65 & 91.0 & 5.830 & 229 \\
288 & 2.15 & 66.0 & 5.710 & 212 \\
303 & 1.78 & 61.0 & 5.648 & 197 \\
318 & 1.54 & 57.8 & 5.441 & 167 \\
333 & 1.32 & 46.2 & 5.210 & 139 \\
\hline
\end{tabular}

\section{Conclusion}

By changing the solvent medium from aqueous to glycolic, the kinetics of film formation on niobium in 0.1 M EDTA (Sodium salt) have been studied and it is observed that the peak voltage, formation rate, current efficiency are increased but differential field of formation decreased with the glycol content of solution. This can be attributed to the decrease in the dielectric constant of the solution with the increase in glycol content of the solution.

It was also observed that the kinetics were good at low temperatures. It was observed that the formation rate, current efficiency, differential field and break down voltage were found to be increasing linearly with the decrease in temperature. The decrease in the field, formation rate and breakdown voltage with the increase in temperature may be attributed to the decrease in the incorporation of anions into the film and may also be due to the dissolution of the film with the increase in temperature.

\section{Acknowledgement}

The authors are thankful to Nuclear Fuel Complex, Hyderabad for generous help in providing $\mathrm{Nb}$ samples and IICT, Hyderabad for SEM images.

\section{References}

1. Balaji T, Govindaiah R, Sharma M K, Purushotham Y, Kumar A and Prakash T L, Mater Lett., 2002, 56, 560

2. Schmidt P F, Huber F and Schwary R F, J Phys Chem Solids, 1960, 15(3-4), 270-290; DOI:10.1016/0022-3697(60)90251-1

3. Sasaki Y, J Phys Chem Solids, 1960, 13, 177-186; https://doi.org/10.1016/00223697(60)90001-9

4. $\quad$ Rupprecht J, Naturwiss, 1960, 47, 127.

5. Aladjem A, J Mater Sci., 1973, 8(5), 688-704; DOI:10.1007/BF00561225

6. Lavanya A and Anjaneyulu Ch, Bull Electrochem., 2002, 18, 317-320.

7. Lavanya A, Sarma C S N and Anjaneyulu Ch, J Electrochem Soc India, 2001, 50, 164.

8. Vermilyea D A, Acta Met., 1953, 1(3), 282-291; DOI:10.1016/0001-6160(53)90101-1

9. Vermilyea D A, Acta Met., 1954, 2(3), 482-486; DOI:10.1016/0001-6160(54)90070-X

10. Nageshwar Rao B, Venkata Ramana V and Anjaneyulu Ch, J Electrochem Soc India 1996, 45, 27.

11. Mashashi Koyama, Rikagaku Kemyusho Hokoku, 1962, 38(321), 546.

12. Wei Wei, Jan M Macak, Nabeen, Shreshta K and Patrick Schmuki, J Electrochem Soc., 2009, 156(6), K104-K109; DOI:10.1149/1.3116243 
13. Schmidt H K, Capellodes $\mathrm{R}$ and Vidal M I, Vida M T, Lab Invest Components, Electron S A, Fr Rev Tech Thomson C S F., 1982, 14, 657 (FR).

14. Lavanya G, Raghunathareddy G and Anjaneyulu C H, Bulletin of Electrochem., 2004, 20(7), 305-308

15. Panasa Reddy A, Anjaneyulu Ch and Sastry K S, J Electrochem Soc India, 1990, 39, 183.

16. Lavanya A, Sarma C S N and Anjaneyulu Ch, J Electrochem Soc India, 2001,50, 164.

17. Panasa Reddy A, Chary A N, Anjaneyulu Ch and Sastry K S, J Electrochem Soc India, 1984, 33, 229.

18. Shukla V D and Anjaneyulu Ch, J Chem., 2011, 8(1), 71-76; DOI:10.1155/2011/319012

19. Colton J B, Trans Int Chem Engg., 1963, 41, 11.

20. Bhaskar Reddy P and Panasa Reddy A, Bull Electrochem., 2003, 19(11), 481-482.

21. Abdel Rehim S S, Hassan H H and Amin M A, J Applied Electrochem., 2002, 32(11), 1257-1264; DOI:10.1023/A:1021662814303

22. Anjaneyulu Ch and Sastry K S, J Electrochem Soc India., 1981, 30, 282.

23. Panasa Reddy A, Anjaneyulu Ch and Sastry K S, J Electrochem Soc India, 1990, 39, 183.

24. Panasa Reddy A, Chary A N, Anjaneyulu Ch and Sastry K S, J Electrochem Soc India, 1984, 33, 229.

25. Norman J E, Corros Sci., 1977, 17(1), 39-47; DOI:10.1016/0010-938X(77)90038-5

26. Tomashov N P, Chin-Min Shih and Zalivalon F P, Zh Prikl Khim., 1963, 36, 1503. 